\title{
Synthesis of an unusual polar glycopeptidolipid in glucose-limited culture of Mycobacterium smegmatis
}

\author{
Anil Kr. Ojha, ${ }^{1,2}$ Saaket Varma ${ }^{1}$ and Dipankar Chatterji ${ }^{1}$
}
1 Molecular Biophysics Unit, Indian Institute of Science, Bangalore, 560012, India
2 Centre for Cellular and Molecular Biology, Uppal Road, Hyderabad. 500007, India

\author{
Author for correspondence: Dipankar Chatterji. Tel: +91 80309 2836. Fax: +91 803600535. \\ e-mail: dipankar@mbu.iisc.ernet.in
}

\begin{abstract}
There has been a general understanding that Mycobacterium smegmatis produces only apolar glycopeptidolipid (GPL), similar in structure to serovar non-specific GPL of Mycobacterium avium. In this study, synthesis of polar GPL in carbon-starved $M$. smegmatis is reported. Mass spectrometric analysis suggests the polar GPL to be a hyperglycosylated species. The earlier structural studies of polar GPLs from $M$. avium have invariably shown the presence of an oligosaccharide appendage to D-allo-Thr. However, a further chemical analysis using $\beta$-elimination of the newly found polar GPL in $M$. smegmatis shows that the molecule still contains a monosaccharide at the D-allo-Thr, thus suggesting a new form of polar GPL.
\end{abstract}

Keywords : polar glycopeptidolipid, carbon starvation

\section{INTRODUCTION}

Studies on starvation physiology of mycobacteria is considered to provide an insight into the long-term persistence of the bacilli, which is an important challenge to the successful therapy of mycobacterial infections (for review see Parrish et al., 1998). Moreover, nutritionally starved bacteria have been demonstrated to bear significant similarity with the natural persistors (Nyka, 1974). In fact, an in vitro starvation model has led to the discovery of at least two genes, sigF (Chen et al., 2000) and relA/spoT (Primm et al., 2000; Ojha et al., 2000) which are involved in the long term persistence of Mycobacterium tuberculosis. With an objective of gaining further insight into the physiological changes that occur during nutritional starvation in mycobacteria, we have studied the change in the profile of cell surface biologically active lipid, glycopeptidolipid (GPL), of Mycobacterium smegmatis in response to carbon starvation. Although there is a variation in the cell surface composition among different species of mycobacteria, the findings reported here may suggest a general mechanism of environmental regulation of cell wall biosynthesis.

Abbreviations: 6-dTal, 6-deoxy talose; ESI-MS, electrospray ionization mass spectrometry; GPL, glycopeptidolipid; MALDI-TOF, matrix assisted laser desorption ionization-time of flight; nsGPL, non-specific GPL.
GPL is an important cell-surface antigen in Mycobacterium avium, Mycobacterium intracellulare and Mycobacterium scrofulaceum. As the nomenclature suggests, GPLs contain a tripeptide-amino alcohol core, D-PheD-allo-Thr-D-Ala-L-alaninol, which is linked with hydroxylated fatty acyl chain at the N-terminal D-Phe through amide linkage (Brennan \& Goren, 1979). The $\mathrm{D}$-allo-Thr and the terminal L-alaninol are further linked with 6-deoxytalose (6-dTal) and O-methyl rhamnose, respectively, to form serovar non-specific GPL (nsGPL) that are found in all species of M. avium complex (Brennan \& Goren, 1979; Belisle \& Brennan, 1989; Aspinall et al., 1995). The nsGPL, also called core GPL, is further glycosylated at $6-\mathrm{dTal}$ to produce serovarspecific polar GPL (Brennan \& Nikaido, 1995) (Fig. 1a). The antigenic polar GPL (serovar specific) has also been associated with the change in colony morphology among natural isolates of $M$. avium. For example, smoothcolony-forming serovar 20 of $M$. avium has polar glycopeptidolipids (Barrow et al., 1980).

GPLs are also an important cell-surface constituent in some fast-growing mycobacteria such as Mycobacterium chelonae, Mycobacterium fortuitum and M. smegmatis (Brennan \& Nikaido, 1995). However, M. smegmatis, a fast-growing non-pathogenic species, has been reported to produce only the apolar nsGPL, structurally similar to those produced by M. avium (Billman-Jacobe et al., 1999; Daffe et al., 1983; Patterson 
(a)

Fatty acyl-CONH-D Phe-D alloThr-D Ala-L alaninyl-O-Rha

(3,4 di-OMe-Rha)

$(2,3,4$ tri-OMe-Rha)

Polar GPLs

apolar GPLs

(b)

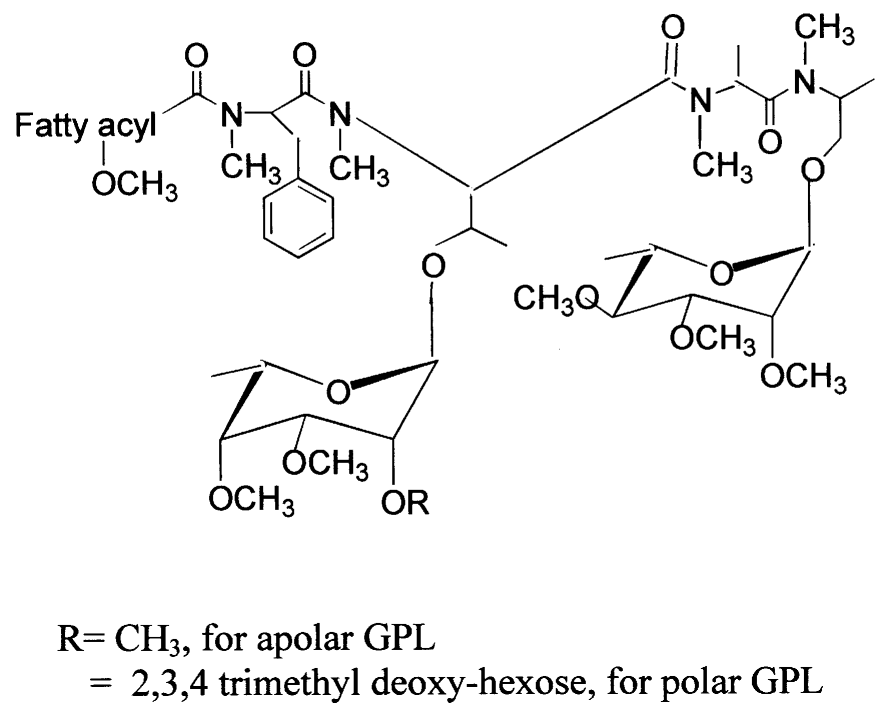

Fig. 1. (a) General structure of mycobacterial GPLs. The arrow heads show further addition of sugars on 6-dTal in polar species of GPLs reported in M. avium. The rhamnose appendage is not found in GPLs from an enriched culture of $M$. smegmatis. (b) The permethylated derivative of GPL.

et al., 2000). Based on the fact that M. smegmatis does not produce multiglycosylated polar GPLs, it has been genetically exploited as a surrogate host for identification of $M$. avium genes encoding specific glycosyltransferases, which add sugars to 6-dTal (Belisle et al., 1991, 1993; Eckstein et al., 1998). Through this strategy a ser 2 locus was identified in $M$. avium that had genes encoding rhamnosyl transferase and fucosyl transferase (Belisle et al., 1991). In this study we report a conditional synthesis of a polar GPL in glucose-limited culture of M. smegmatis. Mass spectrometric analysis suggests that the polar GPL is a hyperglycosylated derivative of the apolar species. Further chemical analysis, however, indicates that the newly found polar species is structurally different from the usual polar GPLs found in $M$. avium in a way that the additional sugar is not linked to 6-dTal.

\section{METHODS}

Bacterial strain, medium and growth. M. smegmatis $\mathrm{mc}^{2} 155$ (Snapper et al. 1990), was used for all experiments. Bacteria were grown in $7 \mathrm{H} 9$ medium supplemented with $2 \%(\mathrm{w} / \mathrm{v})$ glucose and $0.05 \%(\mathrm{v} / \mathrm{v})$ Tween-80. Where necessary, glucose was replaced by $2 \%(\mathrm{v} / \mathrm{v})$ glycerol as an enriched carbon source. For carbon-limited cultures the glucose concentration was reduced to $0.02 \%$ without any change in the concentration of the detergent. For plate culture $1.5 \%$ agar was added to the broth without Tween-80. The concentration of glucose for normal and starved plate cultures were the same as for the respective liqud cultures. For GPL analysis, the bacteria from the preinoculum (fully grown enriched culture) were harvested, washed and then subcultured in the appropriate medium. The growth profile of liquid cultures was obtained by measuring the $\mathrm{OD}_{600}$ at regular time intervals. Colony morphology was observed under the microscope (Zeiss) at $10 \times$ magnification.

Purification and analysis of GPLs. The GPLs from M. smegmatis were purified as mentioned earlier (Khoo et al., 1999). Briefly, lipids were extracted by treating $5 \mathrm{~g}$ (wet wt) cells at various growth phases (see legend for Fig. 3) with chloroform/methanol $(2: 1)$ for $24 \mathrm{~h}$ at room temperature. The organic supernatant was dried and redissolved in chloroform/methanol $(2: 1)$ and treated with an equal volume of $0 \cdot 2 \mathrm{M} \mathrm{NaOH}$ in methanol at $37^{\circ} \mathrm{C}$ for $30 \mathrm{~min}$ then neutralized with a few drops of glacial acetic acid. After drying the solvent lipids were redissolved in chloroform/methanol/water 
$(4: 2: 1)$ and centrifuged. The aqueous layer was discarded and the organic layer containing the lipids was concentrated. The deacylated lipids were spotted on to a silica-coated TLC plate (Merck) and developed with chloroform/methanol $(9: 1)$. The sugar-containing lipids were visualized by spraying the plate with $10 \% \mathrm{H}_{2} \mathrm{SO}_{4}$ (in ethanol) followed by heating at $120{ }^{\circ} \mathrm{C}$ for $10 \mathrm{~min}$. The relative intensity of each spot was determined by densitometric scanning (Bio-Rad) against the plate background.

MS of GPLs. Each species of GPL (marked as spots 1-5) was eluted from a preparative TLC silica plate $(20 \times 20 \mathrm{~cm})$ and dissolved in chloroform/methanol $(2: 1)$. The purity of each species was confirmed by TLC. MALDI-TOF (matrix assisted laser desorption ionization-time of flight; Kratos) was used to obtain the mass of each GPL species of M. smegmatis in positive ion mode using gentisic acid as the matrix. For further analysis of structure, GPLs were permethylated as described earlier (Khoo et al. 1996). Briefly, 0.5 ml DMSO was mixed with one pellet $\mathrm{NaOH}$ and the slurry was added to a vial containing $500 \mu \mathrm{g}$ GPL. To the mixture, $0.5 \mathrm{ml}$ methyl iodide was added and stirred at room temperature for $10 \mathrm{~min}$. The reaction was quenched with slow addition of $1 \mathrm{ml}$ water. The methylated GPL was extracted by adding $2 \mathrm{ml}$ chloroform and washing the chloroform layer three times with water. The organic phase was dried, concentrated and used for MALDI analysis.

$\boldsymbol{\beta}$-Elimination of GPLs. The $\beta$-elimination of sugars from GPLs was carried out as described earlier (McNeil et al., 1989; Eckstein et al., 1998). The purified $\sim 50 \mathrm{mg}$ of each spot (1 and 3) were resuspended in $1 \mathrm{ml}$ absolute ethanol and then $1 \mathrm{ml} 1 \mathrm{M} \mathrm{NaOH}$ was added to it. To each reaction mix, $10 \mathrm{mg}$ $\mathrm{NaBH}_{4}$ was added and the resulting mixture was incubated at $60{ }^{\circ} \mathrm{C}$ for $24 \mathrm{~h}$. Then the reaction mix was cooled to room temperature and neutralized by Dowex $50\left(\mathrm{H}^{+}\right)$resins. Then the solution was filtered and concentrated, and the residue was dissolved in $7 \mathrm{ml}$ chloroform/methanol/water $4: 2: 1$, by vol.). The organic phase containing the lipid fraction was concentrated and analysed by MALDI-TOF. The aqueous layer containing sugar was separated and concentrated. The excess of borate salt was removed by several cycles of coevaporation with methanol. The sugar was peracetylated with $1 \mathrm{ml}$ pyridine/acetic anhydride $(1: 1)$ at $80^{\circ} \mathrm{C}$ for $2 \mathrm{~h}$. The solution was evaporated and the residues were redissolved in acetone and analysed by ESI-MS (electrospray ionization mass spectroscopy; Hewlett Packard) in positive ion mode.

\section{RESULTS}

\section{Glucose-starved $M$. smegmatis forms a smooth colony}

The basis of studying the GPL in glucose-limited culture stems from the observation that there is a marked difference in the colony morphology of M. smegmatis under glucose limitation (Fig. 2). As we can see from Fig. 2 that the M. smegmatis colony grown on $0.02 \%$ glucose assumes a uniform margin, smooth surface and very small size (proportionate to the growth in liquid culture, Table 1) whereas the normal colony of M. smegmatis has non-uniform margin, rugose surface and comparatively very large size. Moreover, the colony on
Table 1. The generation time and stationary phase cell density of $M$. smegmatis as determined from the liquid culture

\begin{tabular}{|lcc|}
\hline $\begin{array}{l}\text { Carbon source } \\
\text { (\% glucose) }\end{array}$ & $\begin{array}{c}\text { Generation } \\
\text { time }(\mathbf{h})^{* *}\end{array}$ & $\begin{array}{c}\text { Maximum cell } \\
\text { density } \dagger\end{array}$ \\
\hline 2 & $2 \cdot 40$ & $5 \cdot 5$ \\
$0 \cdot 2$ & $2 \cdot 73$ & $2 \cdot 7$ \\
$0 \cdot 02$ & $2 \cdot 83$ & $0 \cdot 8$ \\
$0 \cdot 0$ & $4 \cdot 65$ & $0 \cdot 6$ \\
\hline
\end{tabular}

* Calculated as a function of $\mathrm{OD}_{600}$ during the fastest-growing phase of the culture.

$+\mathrm{OD}_{600}$ at stationary phase.
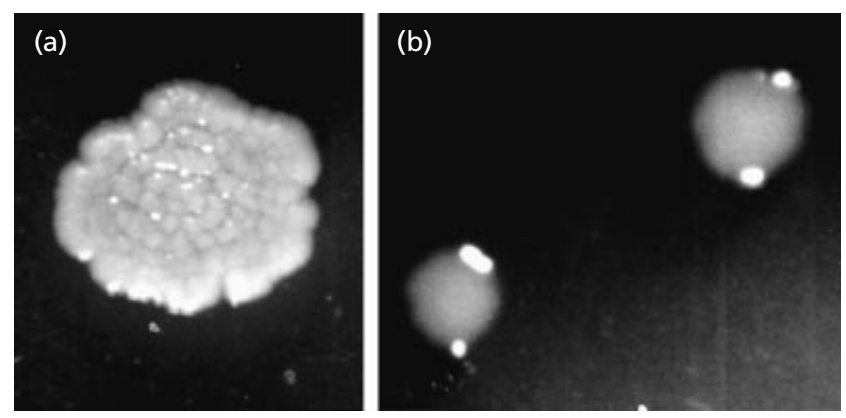

Fig. 2. Comparison of normal ( $2 \%$ glucose) (a) and glucoselimited $(0.02 \%$ glucose $)$ (b) colonies of $M$. smegmatis. Both plates were incubated for one week and photographed at the same magnification.

glucose-limited medium was translucent as against the normal opaque colony.

\section{A polar species of GPL in glucose-limited culture of M. smegmatis}

After observing the changes in colony morphology upon glucose limitation we asked whether it could be translated into variation in the molecular composition of the cell surface. As GPL forms the outermost layer of the cell surface of $M$. smegmatis, we analysed this important cell-surface lipid. Deacylated GPL was isolated from different growth phases of M. smegmatis. Deacylation was carried out using methanolic $\mathrm{NaOH}$ (Brennan \& Goren, 1979). The treatment with methanolic $\mathrm{NaOH}$ esterifies contaminating fatty acyl chains and enriches the alkali-stable GPLs. Fig. 3 shows four major species of apolar GPLs (spots 2-5), observed in normal $(2 \%$ glucose), glucose-limited $(0.02 \%)$ and glucose-starved $(0 \%)$ cultures. The growth of bacteria in the complete absence of glucose is facilitated by the trace amount of carbon source present in the $7 \mathrm{H} 9$ medium and also perhaps from the Tween-80. The GPL profile of the enriched culture is consistent with an earlier one shown by Patterson et al. (2000). Upon comparing the quantity of each spot by densitometric scanning and normalizing 
(a)

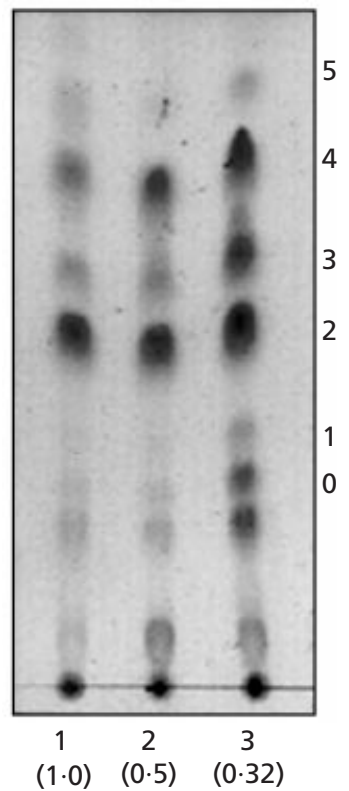

(b)

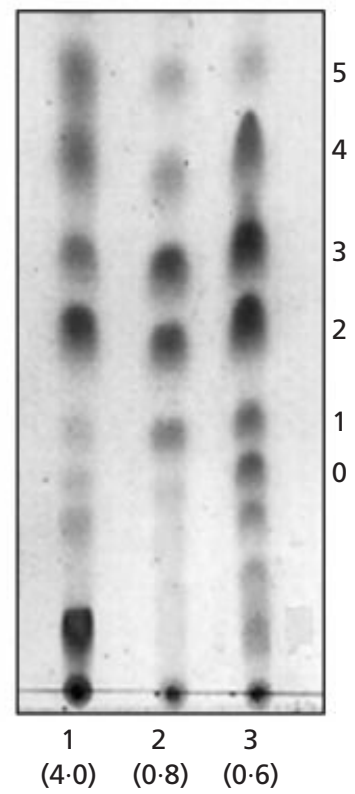

Fig. 3. Profile of GPLs of enriched ( $2 \%$ glucose, lanes 1), glucose-limited $(0.02 \%$ glucose, lanes 2$)$ and glucose-starved $(0.0 \%$ glucose, lanes 3$)$ cultures of $M$. smegmatis identified by TLC on silica-coated plates. The cultures were harvested at two different growth phases, i.e. exponential (a) and stationary phase (b). The $O D_{600}$ reading of each culture at the time of harvest is written below its respective lane. Each spot was assigned the corresponding number based on its mass.

with spot 2, equally present in each sample (data not shown), it was found that glucose-limited bacteria in stationary phase had an additional polar species (spot 1), which was almost absent in actively growing enriched culture. It can be seen from Fig. 3 that the exponential phase of both normal ( $2 \%$ glucose) and glucose-limited $(0.02 \%$ glucose $)$ cultures contain a negligible quantity of the polar species (lanes 1 and 2 of Fig. 3a). The presence of this species is more distinct in stationary phase of the glucose-limited culture (lane 2 of Fig. 3b). The result suggests that the presence of the polar GPL in M. smegmatis is dependent on the concentration of glucose in the medium. To test the conditional synthesis of polar GPLs, we analysed the GPLs of an exponentially growing culture that was completely devoid of glucose $(0 \%$ glucose). As can be seen from lane 3 of Fig. 3a, actively dividing exponential-phase bacteria synthesize polar GPL in the absence of glucose. This observation also rules out the possibility of the growth-phase dependent synthesis of polar GPL, which is also supported by the fact that the stationary phase bacteria from the enriched culture have a very low level of polar GPL. This observation can, once again, be explained by the fact that the presence of polar species is strictly dependent on the concentration of glucose in the medium. Smeulders et al. (1999) have shown that bacteria in an enriched medium enter into the stationary phase at a higher level of carbon source than those grown in carbon-limiting medium. This could perhaps

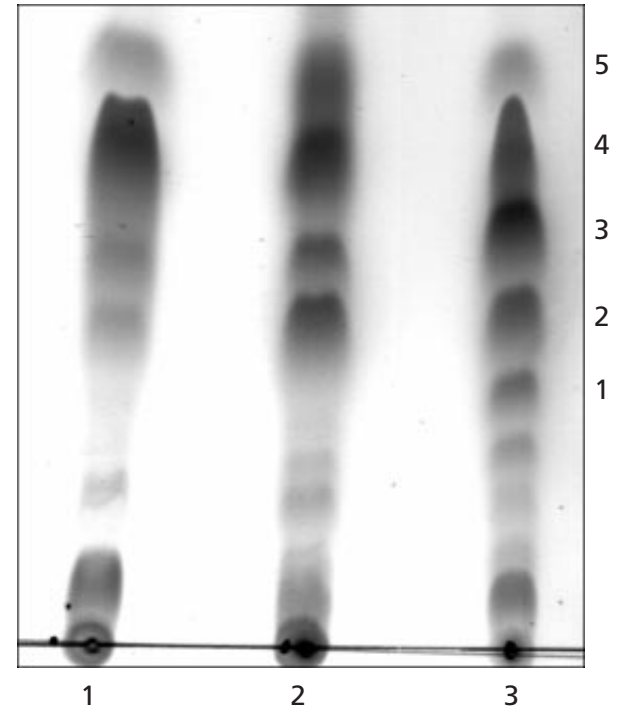

Fig. 4. The synthesis of polar GPL is activated by a lack of carbon source and not derepressed by the absence of glucose. GPL profile of a glycerol-enriched culture $(2 \%)$ in exponential phase (lane 1), is similar to that of a glucose-enriched culture in exponential phase (lane 2). Both the cultures lack the polar species, spot 1, which is found in the GPL profile of carbonstarved ( $0 \%$ glucose) culture (lane 3$)$. The identity of each spot annotated here was confirmed by mass spectrometry.

be due to accumulation of large amounts of growth inhibitory secondary metabolites at very high cell density.

The synthesis of polar species of GPL in glucose-starved medium means that the genetic apparatus for the synthesis of such species is either activated by the lack of a carbon source or derepressed by the lack of glucose (a catabolite repressor). To find out which of the two mechanisms is operating, the GPL profile of a glycerolenriched culture was analysed. Fig. 4 shows that a glycerol-enriched culture doesn't produce the polar species, thus indicating that the synthesis of polar GPL is activated by the lack of carbon source in the medium.

\section{Polar GPL is most likely to be a hyperglycosylated derivative of apolar GPL}

For preliminary identification of the most polar GPL, spot 1, MS of each spot was carried out in native and permethylated (Fig. 1b) state. The mass increment due to permethylation would unambiguously indicate the numbers of free $\mathrm{OH}$ groups that have undergone methyl substitution. Fig. $5 \mathrm{a}$ and $\mathrm{b}$ shows the $m / z$ values of GPLs in native and permethylated forms, respectively. The analysis of mass spectra of apolar GPLs (spots 2-5) was carried out on the basis of the structural studies reported earlier (Billman-Jacobe et al., 1999; Daffe et al., 1983; 
Polar GPL in M. smegmatis

Table 2. $\mathrm{m} / \mathrm{z}$ values of the major molecular ion peak of each GPL spot in native and permethylated states

\begin{tabular}{|ccccc|}
\hline $\begin{array}{l}\text { Spot } \\
\text { no. }\end{array}$ & $\begin{array}{c}\boldsymbol{m} / \boldsymbol{z} \text { of native } \\
\text { GPL }\end{array}$ & $\begin{array}{c}\boldsymbol{m} / \boldsymbol{z} \text { of } \\
\text { permethylated } \\
\text { GPL* }\end{array}$ & $\begin{array}{c}\text { No. methyl groups } \\
\text { transferred }\end{array}$ & $\begin{array}{c}\text { No. free OH } \\
\text { groups }\end{array}$ \\
\hline 1 & 1334 & 1462 & 9 & 5 \\
2 & 1160 & 1286 & 9 & 5 \\
3 & 1174 & 1286 & 8 & 4 \\
4 & 1174 & 1286 & 7 & 3 \\
5 & 1188 & 1286 & 7 & 3 \\
\hline
\end{tabular}

* The $m / z$ values obtained in Fig. 5 are approximated within the error range of the instrument $(0 \cdot 1 \%)$.

† Free $\mathrm{OH}$ groups on the sugars and fatty acyl chain. The free $\mathrm{OH}$ groups are the equivalent $\mathrm{O}$-methyl substitution to the sugars and fatty acyl chain after permethylation. The values are obtained by subtracting 4 methyl groups substituted to tetrapeptide core (see Fig. 1b).

Patterson et al., 2000). Structures of the four apolar GPLs from M. smegmatis strain $\mathrm{mc}^{2} 155$ have been fully characterized by Billman-Jacobe et al. (1999) and Patterson et al. (2000) using GC-MS as well as ESI-MS. The findings from their studies are as follows. 1) Spots 4 and 5 have 2,3,4-trimethyl rhamnose whereas spots 2 and 3 have 3,4-dimethyl rhamnose. 2) Spots 3 and 5 have methoxylated while spots 2 and 4 have hydroxylated fatty acyl chains. 3) All the four deacylated GPLs have unsubstituted 6-dTal. Thus, it implies that the combined total number of free $\mathrm{OH}$ groups on the fatty acyl chain and sugars in spots 5, 4, 3 and 2 would be 3, 4, 4 and 5, respectively. Furthermore, the mass of spots 4 and 3 would remain the same, although their structure and thus, polarity would be different. Consistent with their conclusions we find that spot 5, the most apolar species, has combined total number of three free $\mathrm{OH}$ groups on the fatty acyl chain and the sugars (see the last column of Table 2), all accounted for by 6-dTal. Similarly, the mass and the combined total of free $\mathrm{OH}$ groups on the fatty acyl chains and the sugars of spots 4,3 and 2 exactly follow the structures elucidated in the reported studies (Billman-Jacobe et al., 1999; Daffe et al., 1983; Patterson et al., 2000).

Spot 1, which has a mass of 1334 a.m.u., is heavier than spot 5 by 146 a.m.u. and has a combined total of five free $\mathrm{OH}$ groups on the fatty acyl chain and the sugars. The increment in mass and number of free $\mathrm{OH}$ groups, along with the increased polarity of spot 1 , can be easily satisfied when a deoxyhexose (with an appropriate number of methyl substitutions depending on the precursor) is linked to one of the apolar species. However, the position of linkage can either be 6-dTal or the terminal O-methyl rhamnose.

Interestingly, there was at least one more species of polar GPL, spot 0, which had a relative increase in its quantity upon carbon starvation. The preliminary mass spectrometric analysis showed its mass to be 1320 a.m.u., 14 a.m.u. less than the mass of spot 1 (Fig. 5a). This clearly suggested that spot 0 differs from spot 1 by a methyl substitution and thus raises the possibility that more than one species of apolar GPLs are hyperglycosylated. This spot was not analysed further.

\section{The polar species, spot 1 , is structurally different from the usual polar species in other mycobacteria}

The available literature on the structural studies of GPLs indicates that the polar species of the GPLs in $M$. avium invariably have oligosaccharide appendage linked to 6-dTal (Brennan \& Nikaido, 1995; McNeil et al., 1989). Hence, we asked whether the polar species, spot 1 , was also a result of similar linkage, although it was contrary to the idea suggested by Belisle et al. (1991) and Eickstein et al. (1998) that M. smegmatis is a suitable surrogate host for identification of $M$. avium genes which further glycosylate core GPLs at 6-dTal. For that, $\beta$-elimination of the sugar linked to D-allo-Thr in spot 1 was carried out according to the protocol described earlier (McNeil et al., 1989). One of the apolar species, spot 3, known to have a single 6-dTal at D-allo-Thr was used as a control. The eliminated sugar was reduced, peracetylated and subsequently analysed by ESI-MS. Fig. 6 shows that both spots 1 and 3 released 6-dTal which corresponded to $[\mathrm{M}+\mathrm{Na}]^{+}$of 399 and there was no major molecular ion peak corresponding to a disaccharide $\left([\mathrm{M}+\mathrm{Na}]^{+}\right.$of 629 or higher by a unit of 14$)$ in the spectra of sugar released from spot 1 . This was further corroborated by mass spectral analysis of the organic fraction, which contained the O-rhamnosylated lipotetrapeptide. The MALDI-TOF spectrum of spot 1 (Fig. 7a) expectedly showed a molecular ion peak of 1170 a.m.u., which resulted in the loss of 164 a.m.u. due to removal of 6 -dTal. Similarly, spot 3 showed a major peak at 1010 (1174-164) a.m.u., Fig. 7b. The observation that the new sugar is not linked to 6-dTal is also consistent with the genetic data that provides the evidence for the lack of genes responsible for glycosyl transfer to 6-dTal in M. smegmatis and thus, making it a suitable host for the identification of such genes from 
(a)
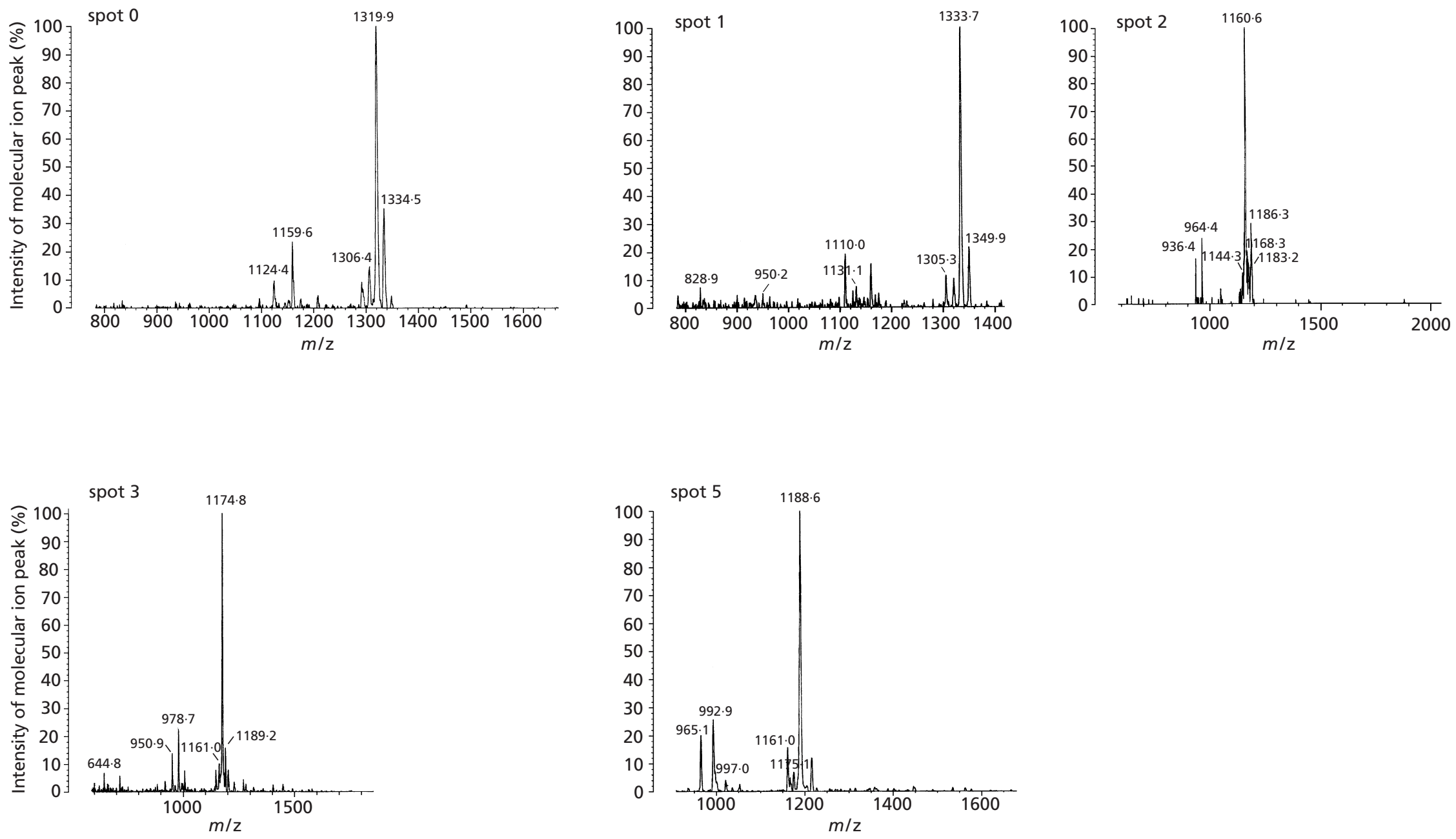
(b)

spot 1

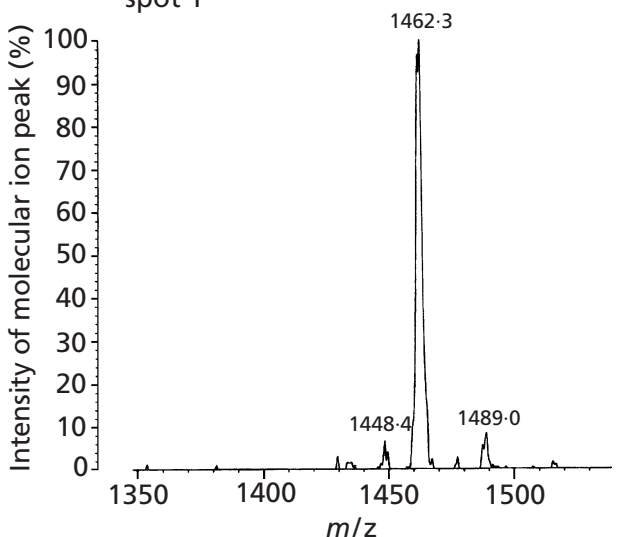

spot 5

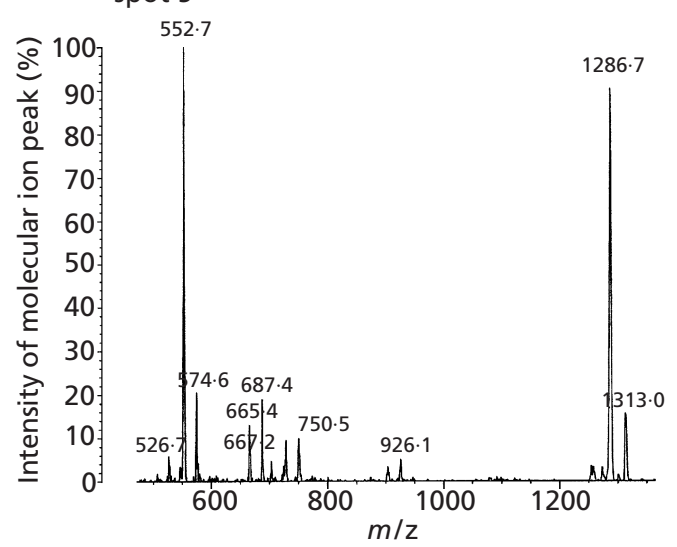

spot 2

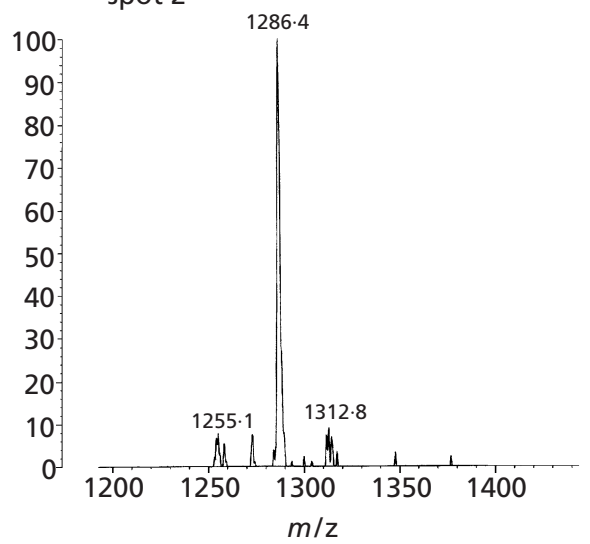

spot 3

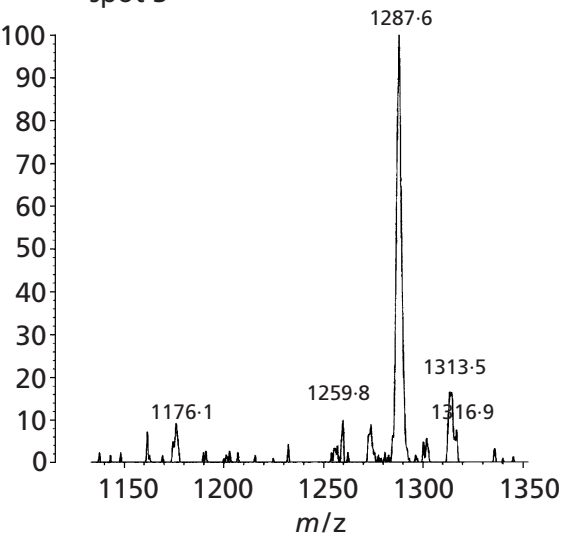

Fig. 5. MS of GPLs from carbon-starved culture of M. smegmatis. (a) Mass spectrum of each species in native state using MALDI-TOF. The spot number is mentioned at the top left of each spectrum. $\mathrm{m} / \mathrm{z}$ value of spot 4 (not shown here) is same as that of spot 3 (see Table 2). (b) Mass spectrum of permethylated derivative of each species using MALDI-TOF. The spot number is mentioned at the top left of each respective spectrum. The $\mathrm{m} / \mathrm{z}$ value of spot 4 (not shown here) is same as that of spot 3 (see Table 2). 
(a)

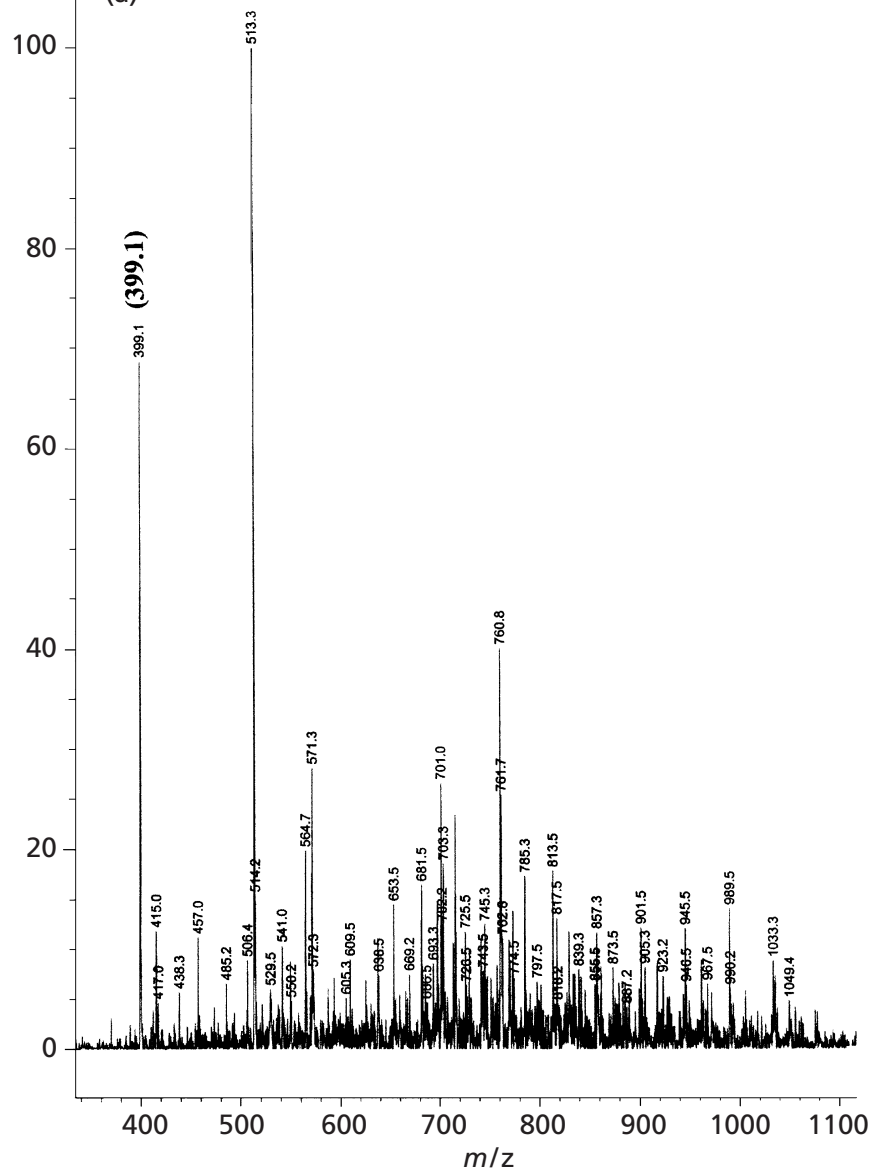

(b)

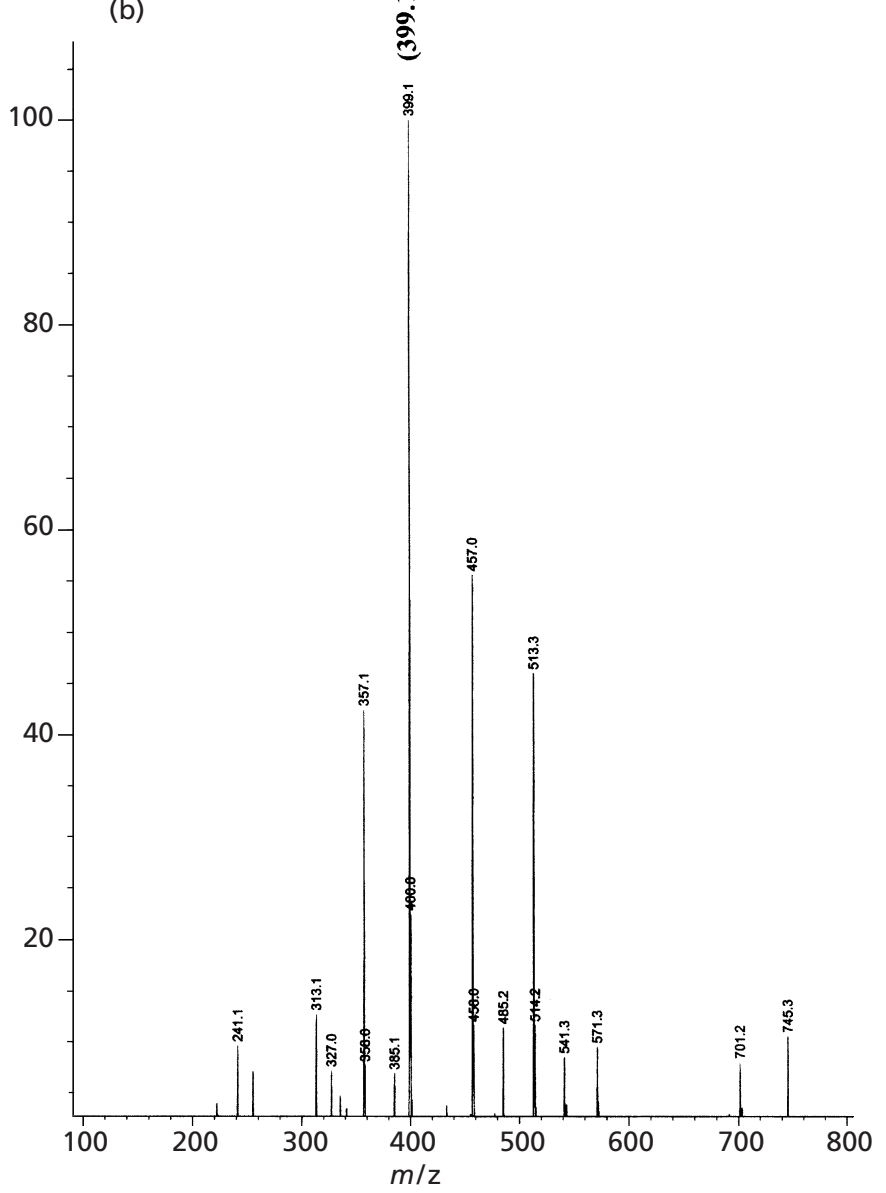

Fig. 6. ESI-MS spectra of the peracetylated derivative of reduced sugar released from spot 1 (a) and spot 3 (b) after $\beta$-elimination. The molecular ion peak of 399 a.m.u. corresponds to the 6-dTal derivative.
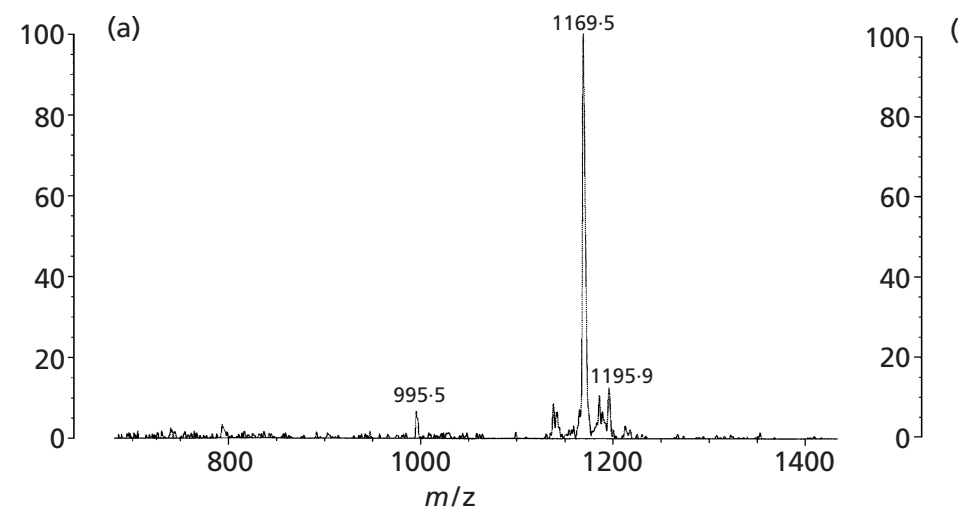

(b)

$1009 \cdot 5$

Fig. 7. MALDI-TOF spectra of the organic fractions, which contained the released lipidic portion of the GPL after $\beta$-elimination of sugars from spot 1 (a) and spot 3 (b). The release of 6 -dTal from the native GPL will decrease the mass of the molecule by 164 a.m.u. 
other species of mycobacteria. Hence, it is very likely that the deoxyhexose may be linked to the terminal rhamnose in the polar species.

\section{DISCUSSION}

The observations reported in this study suggest a possible correlation between the formation of smooth colonies and the synthesis of a polar GPL in M. smegmatis during carbon starvation. Although this study shows that $M$. smegmatis can synthesize polar GPL, the lack of additional sugar at 6-dTal in polar GPL still supports the strategy of using this species as a surrogate host for identifying glycosyltransferases from other species of mycobacteria that transfer sugars to 6-dTal. A comparative analysis of the M. smegmatis genome sequence shows that it contains at least two ORFs (contigs 3314 and 3310 in the TIGR database) that are significantly homologous to the glycosyltransferase gene of M. avium. This suggests that there may be two kinds of glycosylation in M. smegmatis GPL biosynthesis, i.e. a constitutive one for the synthesis of apolar GPLs and a regulated one for the synthesis of polar species. Although the structural finding reveals an unusual polar GPL, evidence for such polar species provides a new insight into the biosynthetic regulation of GPL in M. smegmatis and perhaps also in other mycobacterial species. The detailed structural studies of the polar species and the subsequent identification of genetic apparatus for the synthesis of such species would lead to a better understanding of the molecular basis of the observed phenotype. The involvement of GPL in sliding motility and biofilm formation by M. smegmatis, elegantly studied by Kolter and colleagues (Martinez et al., 1999; Recht et al., 2000; Recht \& Kolter, 2001), demonstrate a wider role of the cell surface lipid in the physiology of the bacteria. In the light of their studies, the regulation of GPL biosynthesis in response to carbon starvation suggests that this cell surface change would be an important adaptive mechanism and may provide a functional advantage to bacilli during such stress. Thus the functional significance of the polar GPL in M. smegmatis would be an obvious follow-up to this study.

\section{ACKNOWLEDGEMENTS}

We thank Professor N. Jayaraman and O. Srinivas for their helpful discussion during the course of this work. We also thank Molecular Biophysics Unit, Indian Institute of Science, Bangalore for the mass spectrometry facility. This work was carried out by the financial aid provided by Department of Biotechnology, Govt of India and Council of Scientific and Industrial Research, Govt of India.

\section{REFERENCES}

Aspinall, G. O., Chatterjee, D. \& Brennan, P. J. (1995). The variable surface glycolipids of mycobacteria: structures, synthesis of epitopes, and biological properties. Adv Carbohydr Chem Biochem 51, 169-242.

Barrow, W. W., Ullom, B. P. \& Brennan, P. J. (1980). Peptidogly- colipid nature of the superficial cell wall sheath of smoothcolony-forming mycobacteria. J Bacteriol 144, 814-822.

Belisle, J. T. \& Brennan, P. J. (1989). Chemical basis of rough and smooth variation in mycobacteria. J Bacteriol 171, 3465-3470.

Belisle, J. T., Pascopella, L., Inamine, J. M., Brennan, P. J. \& Jacobs, W. R., Jr. (1991). Isolation and expression of a gene cluster responsible for biosynthesis of the glycopeptidolipid antigens of Mycobacterium avium. J Bacteriol 173, 6991-6997.

Belisle, J. T., McNeil, M. R., Chattejee, D., Inamine, J. M. \& Brennan, P. J. (1993). Expression of the core lipopeptide of the glycopeptidolipid surface antigens in rough mutants of Mycobacterium avium. J Biol Chem 268, 10510-10516.

Billman-Jacobe, H., McConville, M. J., Haites, R. E., Kovacevic, S. \& Coppel, R. L. (1999). Identification of a peptide synthetase involved in the biosynthesis of glycopeptidolipids of Mycobacterium smegmatis. Mol Microbiol 33, 1244-1253.

Brennan, P. J. \& Goren, M. B. (1979). Structural studies on the type-specific antigens and lipids of the Mycobacterium avium, Mycobacterium intracellulare, Mycobacterium scrofulaceum serocomplex: Mycobacterium intracellulare serotype 9. J Biol Chem 254, 4205-4211.

Brennan, P. J. \& Nikaido, H. (1995). The envelope of mycobacteria. Annu Rev Biochem 64, 29-63.

Chen, P., Ruiz, R. E., Li, Q., Silver, R. F. \& Bishai, W. R. (2000). Construction and characterization of a Mycobacterium tuberculosis mutant lacking the alternate sigma factor gene, sigF. Infect Immun 68, 5575-5580.

Daffe, M., Laneelle, M. A. \& Puzo, G. (1983). Structural elucidation by field desorption and electron-impact mass spectrometry of the C-mycosides isolated from Mycobacterium smegmatis. Biochim Biophys Acta 751, 439-443.

Eckstein, T. M., Silbaq, F. S., Chatterjee, D., Kelly, N. J., Brennan, P. J. \& Belisle, J. T. (1998). Identification and recombinant expression of a Mycobacterium avium rhamnosyltransferase gene $(r t f A)$ involved in glycopeptidolipid biosynthesis. J Bacteriol 180, 5567-5573.

Khoo, K. H., Chatterjee, D., Dell, A., Morris, H. R., Brennan, P. J. \& Draper, P. (1996). Novel O-methylated terminal glucuronic acid characterizes the polar glycopeptidolipids of Mycobacterium habana strain TMC $5135 \mathrm{~J} \mathrm{Biol} \mathrm{Chem} \mathrm{271,} \mathrm{12333-12342.}$

Khoo, K. H., Jarboe, E., Barker, A., Torrelles, J., Kuo, C. W. \& Chatterjee, D. (1999). Altered expression profile of the surface glycopeptidolipids in drug-resistant clinical isolates of Mycobacterium avium complex. J Biol Chem 274, 9778-9785.

Martinez, A., Torello, S. \& Kolter, R. (1999). Sliding motility in mycobacteria. J Bacteriol 181, 7331-7338.

McNeil, M., Chatterjee, D., Hunter, S. W. \& Brennan, P. J. (1989). Mycobacterial glycolipids: isolation, structures, antigenicity, and synthesis of neoantigens. Methods Enzymol 179, 215-242.

Nyka, W. (1974). Studies on the effect of starvation on mycobacteria. Infect Immun 9, 843-850.

Ojha, A. K., Mukherjee, T. K. \& Chatterji, D. (2000). High intracellular level of guanosine tetraphosphate in Mycobacterium smegmatis changes the morphology of the bacterium. Infect Immun 68, 4084-4091.

Parrish, N. M., Dick, J. D. \& Bishai, W. R. (1998). Mechanisms of latency in Mycobacterium tuberculosis. Trends Microbiol 6, 107-112.

Patterson, J. H., McConville, M. J., Haites, R. E., Coppel, R. L. \& Billman-Jacobe, H. (2000). Identification of a methyltransferase from Mycobacterium smegmatis involved in glycopeptidolipid synthesis. J Biol Chem 275, 24900-24906. 
Primm, T. P., Andersen, S. J., Mizrahi, V., Avarbock, D., Rubin, H. \& Barry, C. E., III (2000). The stringent response of Mycobacterium tuberculosis is required for long-term survival. J Bacteriol 182, 4889-4898.

Recht, J. \& Kolter, R. (2001). Glycopeptidolipid acetylation affects sliding motility and biofilm formation in Mycobacterium smegmatis. J Bacteriol 183, 5718-5724.

Recht, J., Martinez, A., Torello, S. \& Kolter, R. (2000). Genetic analysis of sliding motility in Mycobacterium smegmatis. $J$ Bacteriol 182, 4348-4351.
Smeulders, M. J., Keer, J., Speight, R. A. \& Williams, H. D. (1999). Adaptation of Mycobacterium smegmatis to stationary phase. J Bacteriol 181, 270-283.

Snapper, S. B., Melton, R. E., Mustafa, S., Kieser, T. \& Jacobs, W. R., Jr (1990). Isolation and characterization of efficient plasmid transformation mutants of Mycobacterium smegmatis. Mol Microbiol 4, 1911-1919.

Received 10 June 2002; revised 8 July 2002; accepted 12 July 2002. 